\title{
Taking the vehicle out of drug delivery
}

\section{Self-delivery systems for combination therapy}

\author{
Jianliang Shen ${ }^{1}$, Joy Wolfram ${ }^{1}$, Mauro Ferrari ${ }^{1,2}$, Haifa Shen ${ }^{1,3, *}$ \\ 1. Department of Nanomedicine, Houston Methodist Hospital Research Institute, Houston \\ 77030 (USA)
}

2. Department of Medicine, Weill Cornell Medicine, New York 10065 (USA)

3. Department of Cell and Developmental Biology, Weill Cornell Medicine, New York 10065 (USA)

*E-mail: hshen@ @oustonmethodist.org

The biodistribution of drugs has a major impact on therapeutic efficacy and side effects. Previously, nanocarriers have been utilized to improve localized drug delivery, and there are several well-known examples of transport advantages that arise on the nanoscale, including reduced renal clearance and the enhanced permeability and retention (EPR) effect. In addition to improving the biodistribution of drugs, nanocarriers also provide protection from degradation and aid in intracellular uptake. However, a recent literature review of nanoparticle biodistribution profiles found that less than $1 \%$ (median) of systemically injected nanoparticles reach the intended target and the majority of the dose accumulates in healthy tissues [1]. Moreover, the drug concentration that pathological cells are exposed to is further limited by the drug loading capacity of nanocarriers, which is usually around $10 \%$. Indeed, although particles in the nanosize range display favorable transport properties compared to small molecules, the bulk of the injected material lacks therapeutic activity. This excess material could by itself pose a safety concern. In particular, drug delivery vehicles that are not adequately metabolized and eliminated from the body may cause toxicity. Furthermore, the administration of large quantities of foreign material could activate the immune system, potentially leading to increased drug clearance and immunotoxicity. Additionally, it is probable that cells will eventually reach a limit in regards to the uptake capacity of drug carriers. In cases where this internalization limit is reached before the therapeutic threshold of the drug is obtained, the loading capacity will be a major limitation for therapeutic efficacy. These challenges have motivated the development of self-delivery systems that are formed by self-assembly of different therapeutic agents, including chemotherapy, proteins, and small interfering RNA (siRNA) (Fig. 1). This strategy eliminates the need for carriers that lack therapeutic properties and provides an effective means for developing 
synergistic combination therapies, enhancing loading capacity, and exploiting nanoparticle transport properties.

Zhao et al. recently demonstrated that siRNA could self-assemble with polymeric metformin (PolyMet), forming $\sim 80 \mathrm{~nm}$ particles [2]. Although metformin is clinically approved for the treatment of diabetes, this drug has shown promise as an anticancer agent in multiple clinical trials. Moreover, metformin contains guanidine groups, which have previously been shown to increase cellular uptake, enhance transfection, and circumvent efflux pumps [3]. PolyMet was synthesized through the conjugation of linear polyethylenimine (PEI) with dicyandiamide. The amino groups of PEI enabled self-assembly of anionic siRNA molecules with PolyMet (Fig. 1, example 1). Hyaluronic acid (HA), an anionic polysaccharide, was used to condense the nanoparticles. The particles were then coated with a layer of 1,2-dioleoyl-3trimethylammonium-propane chloride (DOTAP) and cholesterol to improve particle stability and siRNA protection. Finally, a pegylated targeting ligand for sigma receptors that are overexpressed on certain cancer cells [4] was added to the particle surface. The therapeutic efficacy of the nanoparticles was assessed in mouse models of non-small cell lung cancer (NSCLC) and melanoma. Vascular endothelial growth factor (VEGF) was selected as the target gene for siRNA therapy. Prior to assessing the anticancer activity of the combination therapy, the effect of metformin nanoparticles on tumor growth was evaluated. The results indicated that nanoparticle-treatment caused a dramatic reduction in tumor growth compared to control groups [2]. Addition of VEGF siRNA to the carrier further enhanced the therapeutic efficacy, leading to a more than twofold and 1.5-fold reduction in tumor growth compared to metformin nanoparticles in a NSCLC and melanoma mouse model, respectively [2]. The nanoparticle platform described herein stands out as an innovative solution to enable efficient combination therapy, since the functionality of the sub-components is maximized. Specifically, polymeric metformin serves both as a therapeutic agent and carrier for siRNA. As a delivery component, polymeric metformin provides siRNA protection and efficient transfection, partially due to the presence of guanidine groups in the drug, which have been found to traverse cell membranes and tissue barriers [3]. It has been speculated that guanidine is able to penetrate the cell membrane by forming a bidentate hydrogen bond with cell surface groups, such as phosphates, carboxylates, and sulfates [3]. Although several studies have previously utilized guanidine-rich carriers for nucleic acid delivery, these carriers lacked therapeutic activity, making them less attractive for multipurpose use.

Another example of a compound that has been used both as a carrier and therapeutic agent is epigallocatechin gallate (EGCG), which is derived from green tea. EGCG has previously been found to inhibit tumor invasion, tumor growth, and angiogenesis [5, 6]. In a study by Chung et $a l$., the ability of EGCG to bind biomolecules was exploited, and $\sim 90 \mathrm{~nm}$ particles were formed through self-assembly of EGCG derivatives with the anticancer proteins Herceptin or interferon alpha-2a (IFN- $\alpha$ 2a) (Fig. 1, example 2) [7]. Specifically, oligomerized EGCG and protein complexation led to the formation of a core complex, which was then assembled with EGCG-PEG to form a stealth shell. Notably, the nanoparticles remained stable in serum and 
were able to protect the protein from enzymatic degradation. It was shown that protein activity was restored upon disassociation from the nanoparticles, an event that takes place through hydrophobic competition with endogenous amphiphilic molecules, such as lipids in the cell membrane. The results from the study revealed that the blood circulation half-life of Herceptin and IFN- $\alpha$ 2a was substantially increased (29-fold) as a result of self-assembly with EGCG [7]. The therapeutic efficacy of the nanoparticles was evaluated in cell culture and in animal models of cancer. In the case of both proteins, a synergistic anticancer effect was observed in response to combination therapy with EGCG (combination index $<1$ ). In a human epidermal growth factor receptor 2 (HER2)-positive breast cancer model, the tumor accumulation of Herceptin nanoparticles increased more than two-fold compared to free Herceptin [7]. Moreover, treatment with EGCG-Herceptin nanoparticles led to a greater reduction in tumor burden compared to sequential treatment with Herceptin and EGCG-albumin nanoparticles. Likewise, in a hepatocellular cancer model, EGCG-IFN- $\alpha$ 2a nanoparticles displayed a more than threefold increase in tumor accumulation, and caused a dramatic reduction in tumor growth compared to free IFN- $\alpha 2 \mathrm{a}$ or sequentially injected IFN- $\alpha 2 \mathrm{a}$ and EGCG-albumin nanoparticles. These observations indicate that EGCG is an efficient carrier for therapeutic proteins. An impressive aspect of the nanoparticles is that they consist entirely of agents with therapeutic activity, with the exception of PEG. In contrast, the previously described PolyMet/siRNA nanoparticles encompass several additional components such as hyaluronic acid, DOTAP, cholesterol, and PEG.

Self-assembly of therapeutic proteins with chemotherapeutic agents has also been reported. Nevala and colleagues recently designed a nanoparticle platform consisting of albumin, paclitaxel, and the therapeutic protein bevacizumab, which blocks VEGF signaling (Fig. 1, example 3) [8]. Self-assembly of Abraxane, a clinically approved nanoparticle consisting of albumin-bound paclitaxel, and bevacizumab led to the formation of stable nanoparticles. Bevacizumab was noncovalently linked to the albumin scaffold of Abraxane. Notably, the size of the nanoparticles ranged from $\sim 160-2170 \mathrm{~nm}$, depending on the concentration of bevacizumab. Studies revealed that the activity of bevacizumab was retained upon self-assembly with Abraxane. The therapeutic efficacy of $160 \mathrm{~nm}$ particles was evaluated in a melanoma mouse model. The results from the study demonstrated that mice treated with Abraxane-bevacizumab nanoparticles had reduced tumor growth and increased survival compared to mice treated with sequential injections of Abraxane and bevacizumab [9]. These results suggest that complex formation between these proteins provides clear therapeutic benefits. Notably, in addition to exerting anticancer activity, bevacizumab also increased the tumor accumulation of abraxane by binding to VEGF in the tumor microenvironment. These promising preclinical findings have led to the initiation of a phase I clinical trial for Abraxane-bevacizumab nanoparticles [9].

A fourth example of a self-assembly strategy to form self-delivery systems for combination therapy is based on the clinically approved anticancer drugs irinotecan and chlorambucil [10]. Irinotecan is a hydrophilic DNA topoisomerase I inhibitor, while chlorambucil is a hydrophobic DNA-alkylating agent. Nanoparticles can be formed through 
self-assembly of amphiphilic irinotecan-chlorambucil drug conjugates in aqueous solution (Fig. 1, example 4). The self-assembly process proceeds through the secondary aggregation of micelles, leading to the formation of $\sim 80 \mathrm{~nm}$ particles. The drug conjugates are linked through an ester bond that can be cleaved in tumor cells. Studies evaluating the performance of these nanoparticles have revealed that they remain stable when exposed to plasma proteins and have a blood concentration that is 20 times higher than that of the free drugs 12 post-injection [10]. Moreover, in vitro results have demonstrated that the nanoparticles are able to circumvent $\mathrm{p}$ glycoprotein-mediated drug resistance. In a mouse model of breast cancer, treatment with irinotecan-chlorambucil nanoparticles led to decreased tumor volume and tumor weight compared to combination therapy with free drugs. For example, after 24 days, the mean tumor volume in the nanoparticle and free drug combination groups was $\sim 32 \%$ and $\sim 58 \%$ of control tumors, respectively. This self-delivery strategy opens up opportunities for the design of various self-assembled nanoparticles based on amphiphilic drug-drug conjugates.

In summary, the self-delivery of combination therapies provides a means for exploiting nanoscale transport characteristics without the use of excess materials that lack therapeutic efficacy. These strategies pave the way for a next generation of therapeutic nanoparticles for the treatment of disease.

\section{Acknowledgements}

This work was funded by the Houston Methodist Research Institute. Partial funds were acquired from: the Ernest Cockrell Jr. Distinguished Endowed Chair (M.F.), the US Department of Defense (W81XWH-12-1-0414) (M.F.), the National Institute of Health (1R01CA193880-01A1) (H.S.), (U54CA210181) (M.F.).

\section{Conflict of interest}

The authors have declared that no conflicts of interest exist.

\section{Author information}

Jianliang Shen and Joy Wolfram contributed equally. The authors declare no competing financial interest. All authors have given approval to the final version of this manuscript.

\section{Further reading}

[1] S. Wilhelm, A.J. Tavares, Q. Dai, S. Ohta, J. Audet, H.F. Dvorak, W.C. Chan, Nature Reviews Materials, 1 (2016) 16014.

[2] Y. Zhao, W. Wang, S. Guo, Y. Wang, L. Miao, Y. Xiong, L. Huang, Nature communications, 7 (2016).

[3] J.R. Vargas, E.G. Stanzl, N.N. Teng, P.A. Wender, Molecular pharmaceutics, 11 (2014) 2553-2565.

[4] B.J. Vilner, C.S. John, W.D. Bowen, Cancer Res., 55 (1995) 408-413. 
[5] S. Liao, Y. Umekita, J. Guo, J.M. Kokontis, R.A. Hiipakka, Cancer letters, 96 (1995) 239243.

[6] Y.D. Jung, L.M. Ellis, International journal of experimental pathology, 82 (2001) 309-316.

[7] J.E. Chung, S. Tan, S.J. Gao, N. Yongvongsoontorn, S.H. Kim, J.H. Lee, H.S. Choi, H. Yano, L. Zhuo, M. Kurisawa, J.Y. Ying, Nat Nano, 9 (2014) 907-912.

[8] W.K. Nevala, S.A. Buhrow, D.J. Knauer, J.M. Reid, E.A. Atanasova, S.N. Markovic, Cancer research, (2016) canres. 3131.2015.

[9] M. Green, G. Manikhas, S. Orlov, B. Afanasyev, A. Makhson, P. Bhar, M. Hawkins, Annals of Oncology, 17 (2006) 1263-1268.

[10] P. Huang, D. Wang, Y. Su, W. Huang, Y. Zhou, D. Cui, X. Zhu, D. Yan, Journal of the American Chemical Society, 136 (2014) 11748-11756. 